\title{
SEIZURES IN THE CONTEXT OF OCCULT CEREBROVASCULAR DISEASE
}

Hedley C.A. Emsley ${ }^{1,2,3^{*}}$ and Laura M. Parkes ${ }^{3}$

${ }^{1}$ Department of Neurology, Royal Preston Hospital, Lancashire Teaching Hospitals NHS Foundation Trust, Sharoe Green Lane, Fulwood, Preston, PR2 9HT, UK

${ }^{2}$ Lancaster Medical School, Lancaster University, Bailrigg, Lancaster, LA1 4YW, UK

${ }^{3}$ Division of Neuroscience and Experimental Psychology, Faculty of Biology, Medicine and Health, University of Manchester, Stopford Building, Oxford Road, Manchester, M13 9BG, UK

*Corresponding author hedley.emsley@lancaster.ac.uk

\section{Highlights}

- Occult cerebrovascular disease is an important cause of late-onset seizures and late-onset epilepsy

- LOS/LOE are associated with a three-fold increased risk of stroke

- 'Late-onset' should probably be regarded as being from the fourth decade of life onwards

- There may be a disproportionately increased risk of ICH after LOS/LOE

- There is a bidirectional relationship between LOE/LOS and cognitive impairment/dementia

- There is a pressing need for further research in this field, including epidemiological, natural history and mechanistic studies

\begin{abstract}
There is an important bidirectional relationship between seizures and cerebrovascular disease. Occult cerebrovascular disease (CVD) is an important cause of late-onset seizures (LOS) and lateonset epilepsy (LOE). LOS/LOE are associated with a three-fold increased risk of subsequent clinical stroke. This relationship exists not only in later life, but with 'late-onset' seizures or epilepsy occurring from the fourth decade of life onwards. There is an increasing evidence for the importance of hypertension and cerebral small vessel disease in epileptogenesis, but there is a considerable need for further work to elucidate underlying mechanisms. There may be a disproportionately increased risk of intracerebral haemorrhage after LOS/LOE; this too requires further study. There is also a bidirectional relationship between LOS/LOE and cognitive impairment/dementia: it is likely that there are important interactions between vascular and neurodegenerative pathological processes mediating LOE, stroke and dementia. There is a pressing need for better epidemiological and natural history data as well as elucidation of epileptogenic mechanisms, in order to progress our understanding and to better inform clinical practice.
\end{abstract}

\section{Keywords}

Late-onset seizures; late-onset epilepsy; cerebrovascular disease; small vessel disease; epileptogenesis

This work did not receive any specific grant from funding agencies in the public, commercial, or notfor-profit sectors. 


\section{Background}

Seizures can complicate clinically overt stroke. These can occur as early (acute symptomatic) poststroke seizures, or as late post-stroke seizures, the latter constituting post-stroke epilepsy (PSE) on account of the probability of further seizures being at least $60 \%$ over the subsequent 10 years [1], and therefore fulfilling the criteria for epilepsy [2]. Tacit consensus has effectively now been reached that seizures occurring within one week of stroke are defined as acute symptomatic seizures, and those occurring after one week are regarded as late post-stroke seizures [3]. However, seizures can also arise as a result of otherwise occult cerebrovascular disease (CVD) and can be associated with a significantly increased stroke risk. 'Occult' in this context is used to refer to CVD that has not otherwise expressed itself in the conventional manner as transient ischaemic attack, stroke, or other recognisable presentations of CVD.

There is therefore a complex interface between CVD and epilepsy. There is, in effect, a bidirectional relationship between the two conditions, with clinical stroke being known to predispose to epilepsy, as well as seizures in the absence of prior clinical stroke being associated with significantly elevated stroke risk. It should however be borne in mind that the concept of 'vascular precursor epilepsy' is not new, and dates back perhaps even as far as Hippocrates, who reportedly recognised that seizures "in old people lead to death, or to paralysis, ... because, the vessels are exhausted, and the blood diminished or diluted" [4].

Late-onset epilepsy (LOE) is often defined in more recent literature from the past one or two decades as having its onset after 60 years of age. It is worth noting that the older literature, in fact dating back more than a century, has drawn attention to the need to consider vascular causes for new onset epilepsy from 30 years of age onwards. This point has also been eloquently made by Trinka and colleagues, who summarised the relevant historical literature [4]. CVD is certainly now widely believed to be an important cause of late-onset seizures (LOS)/LOE. LOS and LOE confer an increased risk of subsequent stroke. The annual incidence of epilepsy rises from 86 per 100,000 people aged 65-69 to 135 per 100,000 people aged over 80 [5]. Despite the growing disease burden of LOE and problems associated with it, the perceived importance of LOE appears relatively low, perhaps because seizures in older adults are often controllable with AEDs more easily than in younger adults [6].

Notwithstanding the existing epidemiological data, the true incidence and prevalence of LOS/LOE are difficult to establish reliably. Difficulties include the numerous diagnostic issues. Seizures/epilepsy are the commonest alternative diagnosis among patients initially suspected to have stroke or TIA, and seizures account for $20 \%$ of non-stroke (or non-TIA) diagnoses among 
patients initially suspected to have stroke or TIA [7]. Clinical presentations of seizures in later life are very varied, and can include falls, confusional states, amnesia and focal neurological symptoms [8]. Co-existent cognitive impairment, coupled with the lack of a witness description when the patient lives alone, as well as numerous other factors, can all complicate the assessment.

\section{Evidence from clinical studies}

The first large-scale epidemiological evidence that LOS predict stroke came from a study based on the UK General Practice Research Database (UKGPRD) and published in 2004 [9]. The authors asked whether the onset of seizures after the age of 60 years with no history of overt stroke is associated with an increased risk of subsequent stroke. Using data on 4,709 individuals who had seizures beginning at or after the age of 60 years, and randomly selected matched controls, they found a highly significant difference in stroke-free survival between the two groups $(p<0.0001)$. The relative hazard of stroke at any point for people with seizures compared with the control group was 2.89 (95\% Cl 2.45-3.41).

We previously reviewed the available evidence from clinical studies supporting a relationship between LOE and occult CVD [10]. In summary, we found evidence for (1) a relationship between vascular risk factors and the risk of LOE, apart from the relationship that exists through clinically overt stroke, in patients older than 40 years, (2) an excess of clinically unsuspected, radiological CVD, particularly cortical infarction but apparently also excess small vessel disease (SVD) changes, and (3) an excess (almost threefold) risk of stroke in patients with LOS/LOE.

More recently, several large population-based and/or registry-based studies of LOS and LOE conducted in Taiwan, the US and Sweden have been published. Using Taiwan National Health Insurance claims data, Chang et al. conducted a study of 3,812 patients newly diagnosed with epilepsy in 2000-2008 and 15,248 non-epilepsy comparisons, frequency matched according to sex, age and index year [11]. They compared subsequent stroke diagnoses in both cohorts until the end of 2009, with incidence rates and hazard ratios of stroke being estimated based on sex, age, the average defined daily doses (DDDs) of antiepileptic drugs (AEDs), and comorbidity. The stroke incidence of the epilepsy cohort was 3-fold higher than that of the comparison cohort. The agespecific results indicated that in the epilepsy cohort and the comparison cohort, the risk was highest for the youngest group (20-39 years). Patients with epilepsy exhibited a higher incidence of stroke than did the general population, with the adjusted hazard ratio for stroke among patients with epilepsy being 2.92 (95\% Cls 2.58 to 3.30), strikingly similar to the earlier UKGPRD study [9]. In 
addition, younger patients with epilepsy and patients who took high doses of AEDs exhibited a high risk of stroke.

The risk of stroke in people developing epilepsy aged 60 or older has been well documented, as described above. Stroke incidence in people with epilepsy aged 35-60 was further investigated using South Carolina hospital discharge and emergency department (ED) data from 2000 to 2011 [12]. These investigators studied 21,035 cases with epilepsy and 16,638 controls. Stroke incidence was found to be 2.5 times higher following adult-onset epilepsy (6.3\%) compared with controls (2.5\%). After adjusting for comorbidities and other factors, cases with epilepsy showed a $60 \%$ higher risk of stroke (HR 1.6, 95\% Cls 1.42 to 1.80 ) by comparison with controls. Nearly half of the strokes in cases with epilepsy occurred in those with their first diagnosis made between the ages of 35 and 55 . These findings suggest that adult-onset epilepsy after age 35 warrants consideration of occult CVD as the epilepsy aetiology, which may also increase the risk of subsequent stroke.

Using the Swedish stroke register (Riksstroke), Zelano et al. identified all patients over 60 years of age with first-ever stroke in 2005-2010 ( $n=92,596)$, and obtained cross-sectional data on those with a history of a first seizure or epilepsy diagnosis in the ten years preceding stroke [13]. 1,372 patients $(1.48 \%)$ had a first seizure or epilepsy diagnosis registered less than 10 years prior to the index stroke. This study included an analysis of pathological stroke subtype, and the authors found a consistently higher proportion of intracerebral haemorrhage (ICH) among stroke cases preceded by seizures than infarction across the age strata, with the percentage of $\mathrm{ICH}$ versus infarction ranging from $2.99 \%$ vs $1.90 \%$ among those aged $60-69$ to $0.99 \%$ vs $0.85 \%$ among those aged $90-99$ at the time of stroke. The mean latency between seizure and stroke was 1,474 days (SD 1,209 days). Seizures or epilepsy preceded $1.48 \%$ of stroke in patients older than 60 years of age. Based on recent national incidence figures in Sweden, the authors determined that $5-20 \%$ of incident cases of seizures or epilepsy after 60 years of age could herald stroke, depending on age group. Clearly, these proportions are of a magnitude that merit further study on how to reduce the risk of stroke in patients with LOS/LOE.

\section{Management of stroke risk and the need for additional evidence}

Given the available clinical evidence, it has been argued that LOS should be regarded as a subclinical cerebrovascular disorder [14]. Some national clinical guidelines now recommend screening for, and treating, cerebrovascular disease risk factors in older people with new-onset seizures [15]. There is certainly clear evidence that CVD often underlies otherwise unexplained LOS. However, this is a 
comparatively recent view, and requires further research. There are a number of pressing issues relevant to clinical practice that are deserving of more attention. There is a lack of robust epidemiological data. It certainly seems reasonable to consider patients with a first-ever late-onset seizure and no previous clinically overt CVD to be at increased risk of stroke, and that they may benefit from a diagnostic work-up to identify modifiable vascular risk factors. But further studies are needed to elucidate the risk-benefit ratio of vascular prevention, for instance with regard to the use of anti-platelet agents in such patients, before widespread change in clinical practice. Randomised controlled trials, or failing this, large-scale prospective registry-based studies, are required to adequately inform clinical practice.

The need to address vascular risk in LOS and LOE has been advocated for many years. The extent to which this is pursued in routine practice is not known. The findings by Wannamaker et al. [12] and others, including historic investigators [4], draw attention to the fact that 'late-onset' seizures accompanied by an apparently increased stroke risk should probably be defined be seizures arising from the fourth decade onwards (rather than 'late-onset' being defined as age 60 or older). There may therefore be potential opportunities for intervention (with regard to vascular risk) in a much larger population than previously recognised. This clearly also increases the need for additional evidence. For example, there is a signal from some of the larger population-based studies, as discussed above, that there may be a disproportionate burden of $\mathrm{ICH}$ among cases of stroke occurring after LOS/LOE. This needs to be confirmed in other studies, but in the meantime the riskbenefit ratio of widespread long-term prescription of antiplatelet drugs, for example, in patients presenting with LOE is unclear. Anecdotally, individual clinicians may already have adopted this in their routine practice, but the need for further research is evident.

There is also a need for further research into the propensity of anti-epileptic drugs (AEDs) to modify vascular risk. We have previously considered this issue [10]. In brief, it is important to note the potential deleterious effects of certain AEDs on vascular risk profile: this is probably most important for the enzyme inducing AEDs. Further study is needed, but we believe that in the context of LOE where occult CVD may be culpable (and perhaps in the wider epilepsy population), it may be preferable to consider the use of modern non-enzyme inducing AEDs to minimize the potential for exacerbation of vascular risk.

\section{Insights from imaging studies}


In a previous study using routine clinical brain imaging, we investigated the prevalence of radiological CVD in patients with LOE over 60 years of age [16]. We undertook a retrospective study of 105 patients with LOE and 105 matched controls. Radiological CVD was present in $65.7 \%$ cases and $33.3 \%$ controls $(p<0.0001$ ), with evidence of radiological large artery disease in $21.9 \%$ cases and $1.9 \%$ controls $(p<0.001)$ and radiological small vessel disease (SVD) in $49.5 \%$ cases and $32.3 \%$ controls $(p<0.05)$. Using a semiquantitative visual rating scale, we found a trend towards a greater severity of SVD changes in cases with LOE. An excess of radiological CVD in LOE was therefore driven, at least in part, by SVD.

The mechanisms of epileptogenesis due to otherwise occult CVD of course remain obscure. Whilst lesions involving the cortex (for example, silent cortical infarcts due to large artery disease) are assumed to be epileptogenic as a result of cortical irritability, clinically occult, largely subcortical CVD (including subcortical SVD) is more difficult to reconcile with epileptogenesis. It is assumed that there must be structural or functional disruption of cortico-cortical or subcortico-cortical circuits. Such lesions may of course not be identifiable on conventional imaging. These might include subtle structural lesions not seen on computed tomography (CT) owing to its lesser sensitivity than magnetic resonance imaging (MRI); although routine MRI sequences may miss some lesions (eg microhaemorrhages may not be identified in the absence of gradient-echo or susceptibility weighted sequences). Conventional clinical imaging sequences generally lack sensitivity to other potentially relevant structural and/or functional changes.

Others have noted the importance of SVD in LOE. Gasparini et al. compared patients with epilepsy associated with leukoaraiosis only (EAL) and patients with epilepsy with a well defined vascular lesion, ie post-stroke epilepsy (PSE) [17]. They included 117 subjects, 58 with PSE and 59 with EAL. On univariate analysis, an abnormal EEG and frontal localisation were associated with a lower EAL probability (odds ratio [OR] $0.36,95 \% \mathrm{Cls} 0.15$ to $0.87, \mathrm{p}=0.02$ and $\mathrm{OR} 0.12,95 \% \mathrm{Cls} 0.04$ to $0.37, \mathrm{p}$ $<0.001$, respectively), while temporal localisation was associated with a higher EAL probability (OR $4.0,95 \% \mathrm{Cls} 1.8$ to $9.0, \mathrm{p}<0.001)$. Multivariate analysis confirmed these associations. Therefore, these authors found that in EAL, temporal lobe epilepsy predominates. The findings show that the causal relationship between overt vascular lesions and epilepsy is not straightforward and that there is a need to consider the role of adjunctive factors.

\section{Mechanisms of epileptogenesis}


CVD may cause a number of changes potentially responsible for epileptogenesis. The neurovascular unit (NVU) comprises endothelial cells, associated blood-brain barrier (BBB) tight junctions, basal lamina, pericytes, and parenchymal cells, including astrocytes and neurons, and in health is associated with normal cell to cell signalling and regulation of BBB function, normal neurovascular coupling and neurotransmitter release-reuptake [18]. CVD involves the loss of regulation of processes such as the normal coupling of neural activity and cerebral blood flow, or transport across the BBB - ie, there may be a disruption of normal NVU function. There may be alterations in cerebral haemodynamics and metabolism. The abnormal cerebral microvasculature and BBB breakdown may promote seizures. Inflammation is also deemed likely to contribute to epileptogenesis [19] and may well also be relevant in the context of CVD and epileptogenesis, although this requires further study. We previously proposed the hypothesis that occult CVD may cause LOE as a consequence of the loss of NVU integrity [20]. We depicted established and possible pathways linking hypertension (as a key cause of cerebral small vessel disease) to epileptogenesis. The role of hypertension-related small vessel disease in adult-onset epilepsy has been highlighted by others in a separate recently published review of current experimental and clinical evidence on both direct and indirect role of hypertension in epileptogenesis [21]. Hypertension of course promotes diffuse cerebral microangiopathy, impairment of cerebral perfusion and endothelial dysfunction/BBB dysfunction and leakage. Downstream consequences of these changes, such as neurovascular uncoupling, excitotoxicity, extravasation of serum proteins and inflammation, may then potentially all contribute to epileptogenesis. We, and others, have subsequently studied various aspects included in this proposed model.

\subsection{Diffuse cerebral microangiopathy}

As an example of a study in which SVD progression was effectively probed for its relationship with epileptogenesis, Izutsu et al. investigated focal hyperintensities on peri-ictal diffusion weighted imaging (DWI) MRI [22]. They conducted a retrospective review of consecutive patients with seizure who completed peri-ictal DWI within 24 hours of seizure onset. Patients were grouped according to the presence or absence of diffusion hyperintense lesions (DHLs) in the region of the perforating arteries. Among 677 patients, 23 patients (3.4\%) had DHLs. Analyses of apparent diffusion coefficient values and fluid attenuated inversion recovery images suggested that DHLs were acute or subacute ischemic lesions that had appeared prior to seizure onset. Patients with DHLs were more likely to be older in age, have atrial fibrillation, and coronary artery disease, and have more severe deep white matter hyperintensity or leukoaraiosis compared to patients without DHLs. DHLs detected on peri- 
ictal DWI may represent incidental acute cerebral microinfarcts in the aging brain, especially in patients with small vessel disease.

\subsection{Structural and physiological changes}

We investigated structural and physiological MRI correlates of occult CVD in patients with LOE (without prior history of transient ischaemic attack or stroke), by comparison with controls [23]. Sixteen patients with LOE (mean age \pm SD: $67.6 \pm 6.5$ years) and 15 age-matched control subjects (65.1 \pm 3.9 years) were included in the analysis. Patients with LOE had significantly lower cortical volume than control subjects $(p=0.02$ ) (localised primarily to the temporal lobes on voxel based analysis [VBA]) and significantly higher white matter hyperintensity $(\mathrm{WMH})$ volume $(p=0.047)$. Baseline whole brain arterial arrival time (AAT), the time taken for endogenously labelled blood to reach the tissue on arterial spin labelling (ASL) MRI, was significantly prolonged in patients with LOE by comparison with controls $(p=0.005)$ (predominantly frontal and temporal lobes on VBA). The observed AAT prolongation may arise as a result of disordered cerebral microvasculature. The findings provide some further insight into the role of occult CVD in the pathophysiology of LOE.

\subsection{BBB dysfunction and leakage.}

We have also undertaken a dynamic contrast-enhanced MRI (DCE-MRI) protocol to compare BBB parameters (gadolinium transfer constant $\mathrm{K}^{\text {trans }}$ and plasma volume $\mathrm{Vp}$ ) between patients with $\mathrm{LOE}$, controls with established CVD and healthy controls (unpublished data). We observed evidence for increased $\mathrm{K}^{\text {trans }}$ in patients with LOE and CVD by comparison with the healthy control group. We are undertaking further analysis of these data.

\section{Cognitive impairment and dementia risk: recent findings}

With an ageing population, there will be a substantial rise in the number of older adults with epilepsy. In developed countries, the highest incidence of epilepsy is already in people over 65. Recent studies show that older people with epilepsy are more likely to suffer from cognitive dysfunction and that there might also be an important bidirectional relationship between epilepsy and dementia [24]. Older adults with epilepsy, whether they developed the condition at a younger age or de novo later in life, exhibit poorer performance across a range of cognitive measures compared to healthy controls and have an increased risk of developing dementia. There has been surprisingly little investigation of cognitive function in late-onset epilepsy. 
There are likely to be important inter-relationships between Alzheimer's disease, epilepsy and CVD. It would appear that several overlapping pathologies can contribute to the development of LOE as well as to the development of dementia. In particular, vascular risk factors are common in people with epilepsy and may represent modifiable risk factors for both the development of dementia and of epileptogenesis. At the current time we do not know whether the more aggressive management of vascular risk factors might improve the control of seizures and/or provide protection against cognitive decline in people with epilepsy. There are many other unanswered questions in this area, also highlighted by Sen et al., such as the extent to which pathological burden (vascular, traumatic, tau or amyloid) in people with epilepsy might interact synergistically with ongoing seizure activity, and how this might lead to progressive worsening of cognitive function [24]. There can be little doubt that there is a pressing need for large-scale patient registries with harmonized systems of cognitive assessment.

\section{Conclusions}

Occult cerebrovascular disease is responsible for a significant proportion of LOS and LOE. The definition of 'late-onset' however, is something of a moot point. In most contemporary literature, 'late-onset' has effectively been used to mean 'in later life'. The historic literature in this field, taken together with findings from more recent work, suggests that occult CVD should be regarded as a potential underlying cause of seizures or epilepsy arising from the fourth decade onwards, thus suggesting the definition of 'late-onset' needs to be revised 'downwards'. There is an increasingly compelling case surrounding the importance of hypertension and SVD in epileptogenesis, but there is a considerable need for further work to elucidate underlying mechanisms. There may be a disproportionately increased risk of intracerebral haemorrhage as opposed to ischaemic stroke among patients developing stroke after LOS/LOE, but again, further research is required. It also seems apparent that cognitive impairment and dementia are more frequent after LOS/LOE than may have been appreciated previously, and it appears likely that there are important interactions between vascular and neurodegenerative pathological processes mediating LOE, stroke and dementia. There is a pressing need for better epidemiological and natural history data as well as improved understanding of mechanisms. There is a pressing need for progress in this field in order to better inform clinical practice. This might be best achieved through the establishment of longitudinal 
or prospective registry-based studies to characterise natural history, mechanisms and management approaches, including the role of vascular 'secondary' prevention.

\section{References}

[1] Hesdorffer DC, Benn EK, Cascino GD, Hauser WA. Is a first acute symptomatic seizure epilepsy? Mortality and risk for recurrent seizure. Epilepsia. 2009 May;50(5):1102-8. doi: 10.1111/j.15281167.2008.01945.x. Epub 2009 Jan 26. PubMed PMID: 19374657.

[2] Fisher RS, Acevedo C, Arzimanoglou A, Bogacz A, Cross JH, Elger CE, Engel J Jr, Forsgren L, French JA, Glynn M, Hesdorffer DC, Lee BI, Mathern GW, Moshé SL, Perucca E, Scheffer IE, Tomson T, Watanabe M, Wiebe S. ILAE official report: a practical clinical definition of epilepsy. Epilepsia. 2014 Apr;55(4):475-82. doi:10.1111/epi.12550. Epub 2014 Apr 14. Review. PubMed PMID: 24730690.

[3] Beghi E, Carpio A, Forsgren L, Hesdorffer DC, Malmgren K, Sander JW, Tomson T, Hauser WA. Recommendation for a definition of acute symptomatic seizure. Epilepsia. 2010 Apr;51(4):671-5. doi: 10.1111/j.1528-1167.2009.02285.x. Epub 2009 Sep 3. Review. PubMed PMID: 19732133.

[4] Trinka E, Krämer G, Werhahn K. Vascular precursor epilepsy - Old wine in new skins? Epilepsy Behav. 2015 Jul;48:103-4. doi: 10.1016/j.yebeh.2015.03.026. Epub 2015 Jun 21. PubMed PMID: 26104488.

[5] Wallace H, Shorvon S, Tallis R. Age-specific incidence and prevalence rates of treated epilepsy in an unselected population of 2,052,922 and age-specific fertility rates of women with epilepsy. Lancet. 1998 Dec 19-26;352(9145):1970-3. PubMed PMID: 9872246.

[6] Ramsay RE, Ruggles K, Slater JD. Effects of age on epilepsy and its treatment. Results from the VA cooperative study. Epilepsia 1994; 35 (Suppl 8):91.

[7] Gibson LM, Whiteley W. The differential diagnosis of suspected stroke: a systematic review. J R Coll Physicians Edinb. 2013;43(2):114-8. doi:10.4997/JRCPE.2013.205. Review. PubMed PMID: 23734351.

[8] Brodie MJ, Elder AT, Kwan P. Epilepsy in later life. Lancet Neurol. 2009 Nov;8(11):1019-30. doi: 10.1016/S1474-4422(09)70240-6. Epub 2009 Oct 1. Review. PubMed PMID: 19800848.

[9] Cleary P, Shorvon S, Tallis R. Late-onset seizures as a predictor of subsequent stroke. Lancet. 2004 Apr 10;363(9416):1184-6. PubMed PMID: 15081649.

[10] Gibson LM, Hanby MF, AI-Bachari SM, Parkes LM, Allan SM, Emsley HC. Late-onset epilepsy and occult cerebrovascular disease. J Cereb Blood Flow Metab. 2014 Apr;34(4):564-70. doi: 10.1038/jcbfm.2014.25. Epub 2014 Feb 12. Review. PubMed PMID: 24517978; PubMed Central PMCID: PMC3982095.

[11] Chang CS, Liao CH, Lin CC, Lane HY, Sung FC, Kao CH. Patients with epilepsy are at an increased risk of subsequent stroke: a population-based cohort study. Seizure. 2014 May;23(5):377-81. doi: 10.1016/j.seizure.2014.02.007. Epub 2014 Feb 22. PubMed PMID: 24630806.

[12] Wannamaker BB, Wilson DA, Malek AM, Selassie AW. Stroke after adult-onset epilepsy: a population-based retrospective cohort study. Epilepsy Behav. 2015 Feb;43:93-9. doi: 10.1016/j.yebeh.2014.11.028. Epub 2015 Jan 7. PubMed PMID:25575071. 
[13] Zelano J, Larsson D, Kumlien E, Åsberg S. Pre-stroke seizures: A nationwide register-based investigation. Seizure. 2017 Jul;49:25-29. doi:10.1016/j.seizure.2017.05.010. Epub 2017 May 17. PubMed PMID: 28544888.

[14] Brigo F, Nardone R. Late-onset seizures: a subclinical cerebrovascular disorder? Expert Rev Neurother. 2017 Aug;17(8):751-753. doi:10.1080/14737175.2017.1332995. Epub 2017 May 25. PubMed PMID: 28521561.

[15] Scottish Intercollegiate Guidelines Network (SIGN). Diagnosis and management of epilepsy in adults. Edinburgh: SIGN; 2018. (SIGN publication no. 143). [September 2018]. Available from URL: http://www.sign.ac.uk

[16] Maxwell H, Hanby M, Parkes LM, Gibson LM, Coutinho C, Emsley HC. Prevalence and subtypes of radiological cerebrovascular disease in late-onset isolated seizures and epilepsy. Clin Neurol Neurosurg. 2013 May;115(5):591-6. doi:10.1016/j.clineuro.2012.07.009. Epub 2012 Jul 26. PubMed PMID: 22840415.

[17] Gasparini S, Ferlazzo E, Beghi E, Sofia V, Mumoli L, Labate A, Cianci V, Fatuzzo D, Bellavia MA, Arcudi L, Russo E, De Sarro G, Gambardella A, Aguglia U. Epilepsy associated with Leukoaraiosis mainly affects temporal lobe: a casual or causal relationship? Epilepsy Res. 2015 Jan;109:1-8. doi:10.1016/j.eplepsyres.2014.10.012. Epub 2014 Oct 28. PubMed PMID: 25524836.

[18] Sweeney MD, Ayyadurai S, Zlokovic BV. Pericytes of the neurovascular unit: key functions and signaling pathways. Nat Neurosci. 2016 May 26;19(6):771-83. doi:10.1038/nn.4288. Review. PubMed PMID: 27227366; PubMed Central PMCID: PMC5745011.

[19] Vezzani A, Friedman A, Dingledine RJ. The role of inflammation in epileptogenesis. Neuropharmacology. 2013 Jun;69:16-24. doi:10.1016/j.neuropharm.2012.04.004. Epub 2012 Apr 13. Review. PubMed PMID:22521336; PubMed Central PMCID: PMC3447120.

[20] Gibson LM, Allan SM, Parkes LM, Emsley HC. Occult cerebrovascular disease and late-onset epilepsy: could loss of neurovascular unit integrity be a viable model? Cardiovasc Psychiatry Neurol. 2011;2011:130406. doi: 10.1155/2011/130406. Epub 2011 Mar 6. PubMed PMID: 21461380; PubMed Central PMCID: PMC3063412.

[21] Gasparini S, Ferlazzo E, Sueri C, Cianci V, Ascoli M, Cavalli SM, Beghi E, Belcastro V, Bianchi A, Benna P, Cantello R, Consoli D, De Falco FA, Di Gennaro G, Gambardella A, Gigli GL, ludice A, Labate A, Michelucci R, Paciaroni M, Palumbo P, Primavera A, Sartucci F, Striano P, Villani F, Russo E, De Sarro G, Aguglia U; Epilepsy Study Group of the Italian Neurological Society. Hypertension, seizures, and epilepsy: a review on pathophysiology and management. Neurol Sci. 2019 May 4. doi: 10.1007/s10072-019-03913-4. [Epub ahead of print] Review. PubMed PMID: 31055731.

[22] Izutsu N, Fujimoto Y, Yamada N, Kajikawa R, Yoshimura K, Nagashima M, Wakayama A, Yoshimine T. Small Hyperintensities in the Area of the Perforating Arteries in Patients with Seizure. Eur Neurol. 2018;79(3-4):221-227. doi: 10.1159/000488673. Epub 2018 Apr 6. PubMed PMID: 29627833.

[23] Hanby MF, Al-Bachari S, Makin F, Vidyasagar R, Parkes LM, Emsley HC. Structural and physiological MRI correlates of occult cerebrovascular disease in late-onset epilepsy. Neuroimage Clin. 2015 Aug 20;9:128-33. doi:10.1016/j.nicl.2015.07.016. eCollection 2015. PubMed PMID: 26413475; PubMed Central PMCID: PMC4556750. 
[24] Sen A, Capelli V, Husain M. Cognition and dementia in older patients with epilepsy. Brain. 2018 Jun 1;141(6):1592-1608. doi: 10.1093/brain/awy022. PubMed PMID: 29506031; PubMed Central PMCID: PMC5972564.

\section{Figure legend}

Figure 1. This schematic depicts the temporal relationships between 'pre-stroke seizures', clinical stroke and 'post-stroke seizures' and the underlying growing burden of cerebrovascular disease over time.

\section{Figure 1}

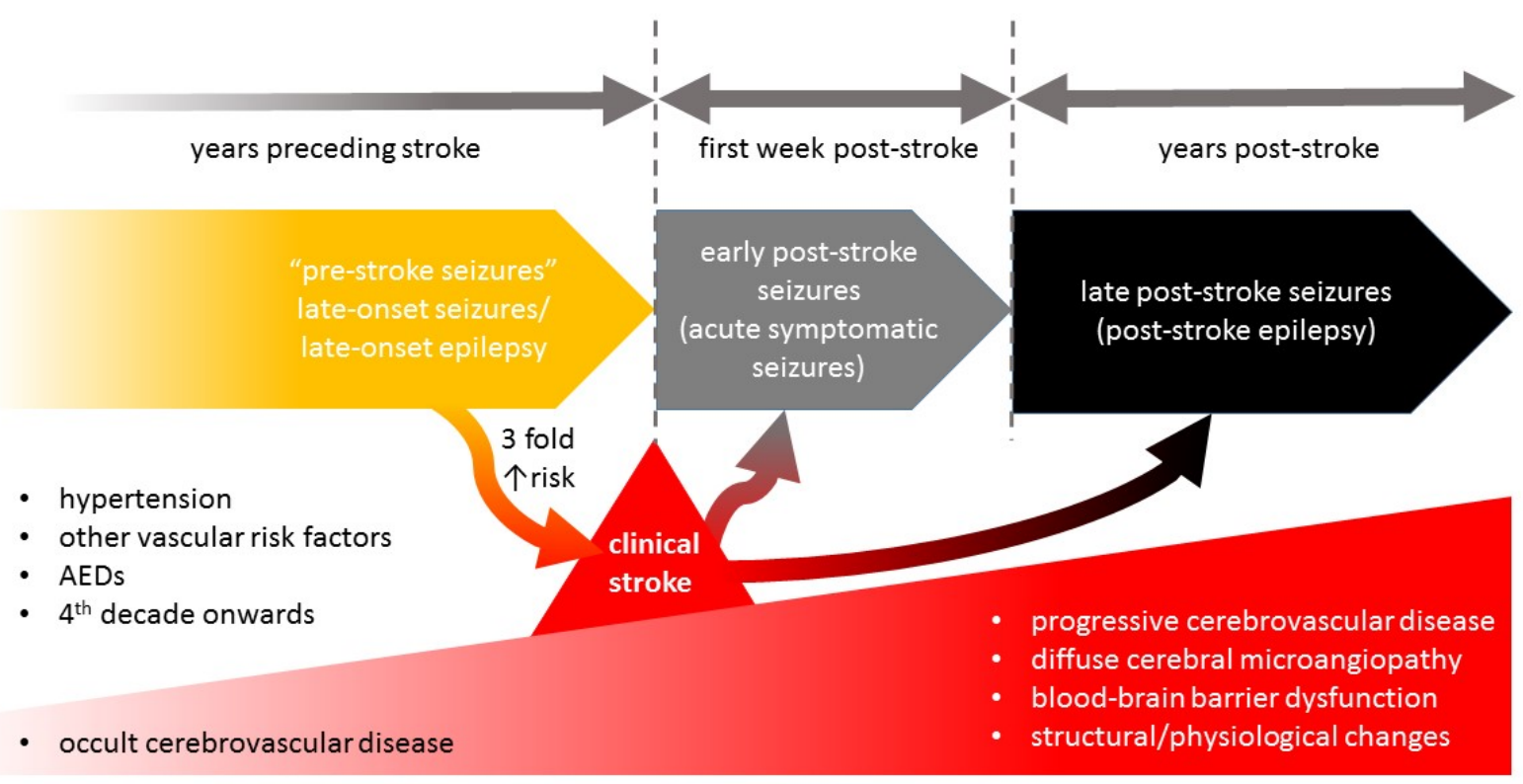

